\title{
Detection of inaccessible head and neck lesions using human saliva and fluorescence spectroscopy
}

\author{
Pavan Kumar ${ }^{1,2}$ iD
}

Received: 14 May 2021 / Accepted: 1 October 2021 / Published online: 12 October 2021

(c) The Author(s), under exclusive licence to Springer-Verlag London Ltd., part of Springer Nature 2021

\begin{abstract}
Head and neck cancer detection using fluorescence spectroscopy from human saliva is reported here. This study has been conducted on squamous cell carcinoma (SCC), and dysplastic (precancer) and control (normal) groups using an in-house developed compact set-up. Fluorescence set-up consists of a 375-nm laser diode and optical components. Spectral bands of flavin adenine dinucleotide (FAD), porphyrins, and Raman are observed in the spectral range of 400 to $800 \mathrm{~nm}$. Presence of FAD and porphyrin bands in human saliva is confirmed by the liquid phantoms of FAD and porphyrin. Significant differences in fluorescence intensities among all the three groups are observed. Three spectral ranges from 455 to 600,605 to 770 , and 400 to $800 \mathrm{~nm}$ are selected for each group and area values under each spectral range are computed. To differentiate among the groups, receiver operating characteristic (ROC) analysis is employed on the area values. ROC differentiates among the groups with accuracies of $98 \%, 92.85 \%$, and $81.13 \%$ respectively in the spectral ranges of 400 to $800 \mathrm{~nm}$. However, in other two spectral ranges ( 455 to 600 and 605 to $770 \mathrm{~nm}$ ), low accuracy values are found. Obtained accuracy values indicate that selection of human saliva for head and neck cancer detection may be a good alternative.
\end{abstract}

Keywords Head and neck cancer $\cdot$ Saliva $\cdot$ Fluorescence $\cdot$ Flavin adenine dinucleotide $\cdot$ Porphyrin $\cdot$ Receiver operating characteristic analysis

\section{Introduction}

Head and neck cancer is the sixth most common malignancy worldwide. In it, different parts of head and neck regions such as oral cavity, salivary glands, pharynx, and larynx are affected. Larynx also called voice box is the most affected site in the neck region. Head and neck cancer cases are due to intake of unhealthy products namely tobacco, betel nuts, cigarette/bidi, and alcohol [1-3]. Poor hygiene and human papillomavirus (HPV) are also responsible. A 5-year survival rate $(\approx 60 \%)$ has not much changed since last few years; therefore, its detection and diagnosis at an early stage is an important concern. If diagnosed at early stage, chances of being cure are significantly higher $(\approx 80 \%)$ [4].

Pavan Kumar

pavan2012iitk@gmail.com

1 Department of Biomedical Engineering, Indian Institute of Technology Ropar, Ropar 140001, India

2 Department of Physics, Indian Institute of Technology Kanpur, Kanpur 208016, India
Conventional biopsy techniques such as brush biopsy, toluidine blue, white light endoscopy, and tissue biopsy are used majorly to identify the head and neck lesions by the pathologist. Among them, tissue biopsy with histopathology is the gold standard [5-7]. Tissue biopsy is an invasive procedure, in which tissue specimen is removed using the surgical blade. Head and neck lesions are usually spread over a large area of a head-neck region. It is therefore difficult to decide best area for biopsy. Due to these complications, patients have to go through multiple biopsies which itself become terrifying. Major complication faced with head and neck patients is the accessibility to the regions (tonsil, larynx, pharynx, etc.). Different non-invasive techniques namely fluorescence, Raman, diffuse reflectance, infrared, OCT, and life time spectroscopy have been used to detect premalignant and malignant lesions of cancer [8-15]. Among them, fluorescence-based spectroscopic and imaging devices have been widely studied by the research groups for the in vivo study. However, Raman and fluorescence-based endoscopy systems have been utilized for larynx cancer detection [16-19]. In the diagnostic media, human tissue is a widely used medium. However, other media such as 
blood, saliva, and urine have also been studied. In recent studies using the spectroscopic devices, body fluids (blood and saliva) have shown promise for the detection of cancer (breast, lung, and oral) [20-23]. As for sampling procedure is concerned, saliva has specific advantages such as easy accessibility as well as ease in sample collection. The average daily production of saliva is 1 to $1.51 \mathrm{in}$ an adult and can be collected multiple times non-invasively from patients and volunteers of any age group. Human saliva consists of several biomarkers such as nucleic acids (DNA and RNA), amino acids (tryptophan, tyrosine, etc.), proteins, amylase, coenzymes (NADH, FAD, etc.), and porphyrins. They have different excitation and emission bands. FAD has emission band near $500 \mathrm{~nm}$ and porphyrin has emission bands at 634 , 676,689 , and $703 \mathrm{~nm}$ respectively. It is well established that concentration of human salivary biomarkers alters with the progression of cancer [24, 25].

Saliva is being accepted as a diagnostic medium for forensic purposes, viz. sexual assault and child abuse cases, drug abuse cases, and for HIV cases [26, 27]. Human saliva has been studied by the Xiaozhou Li et al. group for lung cancer detection using the surface-enhanced Raman spectroscopy system on cancerous and normal saliva samples. An overall decrease in Raman peak intensities was observed in cancerous samples than in the control cases. The group achieved sensitivity of $78 \%$ and specificity of $83 \%$ [22]. M. Yuvaraj et al. studied saliva for oral cancer detection by using excitation and emission fluorescence spectroscopy $(\lambda=405 \mathrm{~nm})$ and reported that porphyrin accumulation in OSCC patients was significantly higher than in the control group. They were able to differentiate OSCC patients from control group with sensitivities of 85.7 and $84.1 \%$ and specificities of 93.3 and 93.2\% [23]. Using the laser-induced fluorescence spectroscopy for the detection of oral cancer on saliva samples, the Patil et al. group differentiated cancerous samples to normal with a sensitivity of $79 \%$ and a specificity of $78 \%$ [28]. Kumar $\mathrm{P}$ et al. studied saliva for oral cancer detection and performed fluorescence $(\lambda=350 \mathrm{~nm})$ and Stokes shift spectroscopy $(\Delta \lambda=120 \mathrm{~nm})$ measurements. Bands of NADH, FAD, and porphyrin were observed in the spectra. They were able to classify the groups (OSCC, OSMF, and normal) with sensitivities and specificities of up to $90 \%$ [29]. Human saliva is also studied for coronavirus detection by the Kai-wang To et al. group and they achieved the accuracy of $91.7 \%$ [30]. The Desai et al. group performed a study on human saliva for RNA virus detection using Raman spectroscopy and achieved sensitivity of $92.5 \%$ and specificity of $88.8 \%$ respectively [31].

Since accessibility to the most of the head and neck regions is a problem except the oral cavity, therefore use of saliva may be more beneficial for its detection. Although oral cavity lesions are clearly visible in patients having difficulty in opening the mouth (i.e. oral submucosal fibrosis
(OSMF)) and patients with lesions deep inside the cavity (i.e., tonsillar fossa), human saliva may be a good substitute. In this study, inaccessible lesions of head and neck patients (larynx, tonsillar fossa, and pharynx) are incorporated. An in-house developed fluorescence spectroscopy system is utilized. Liquid phantoms of flavin adenine dinucleotide (FAD) and porphyrin are prepared and their spectrums are recorded. Three spectral ranges $(455-600 \mathrm{~nm}, 605-770 \mathrm{~nm}$, and $400-800 \mathrm{~nm}$ ) that correspond to FAD band, porphyrin bands, and combination of FAD, porphyrin, and Raman bands of saliva samples are selected respectively. Area under all the three spectral ranges is computed individually. Receiver operating characteristic (ROC) analysis is used to differentiate among the groups. ROC is employed on the area values of each spectral range of all the three groups and diagnostic parameters (sensitivity, specificity, and accuracy) are computed.

\section{Materials and methods}

\section{Sample collection}

Liquid phantoms (Sigma-Aldrich, USA) of $40 \mu \mathrm{M}$ concentration were prepared by using commercially available fluorophores (FAD and porphyrin). Liquid phantoms were prepared and diluted using distilled water. Saliva samples were collected from 31 SCC patients, 22 dysplastic patients, and 20 patients that belong to the control group. The mean age of SCC, dysplastic, and control groups with the standard deviation was $54 \pm 13,46 \pm 9$, and $37 \pm 8$ respectively. Patients were instructed not to consume any food items at least 3 to $4 \mathrm{~h}$ before reporting to clinicians and suggested to rinse their oral cavity to omit the effect of foodstuff in saliva. All the required information of the patients and volunteers (e.g., age, occupation, family background, life style, and habits) were noted. Normal volunteers did not have any habits of consumption of any kind of tobacco products and were free from any disease during the study. Collection of saliva samples was done in Hallet Hospital affiliated to GSVM Medical College, Kanpur. A few milliliters of sample ( -1 to $2 \mathrm{~mL}$ ) from each patient and volunteer were collected in sterile containers. To pursue this work, ethical clearance was obtained.

\section{Instrumentation}

For the fluorescence measurements, a compact system was developed in the lab. Schematic diagram of the setup with the required accessories is shown in Fig. 1. It consists of a 375-nm laser diode, cuvette holder, and optical components, viz. collimating lenses (CL), long pass filter (LPF), and connecting fibers. In the cuvette holder, two collimating 


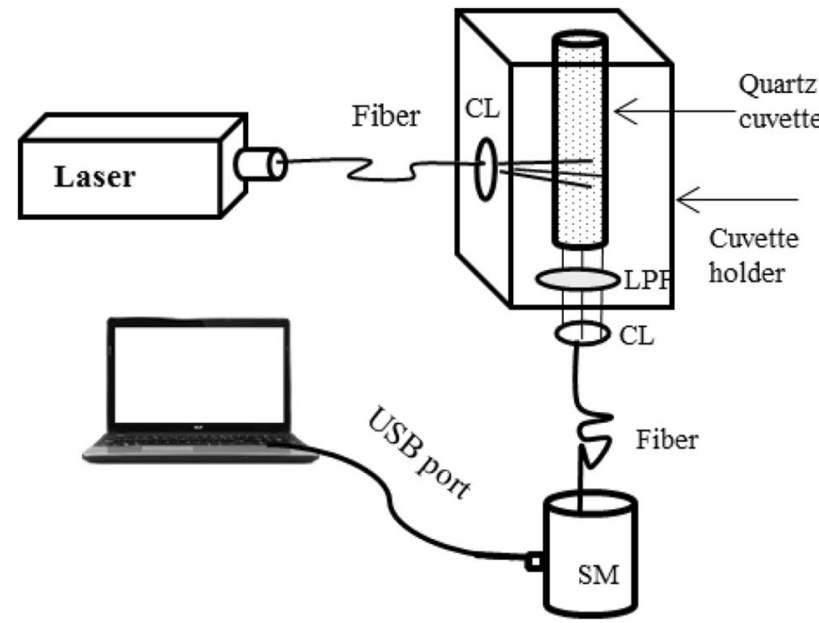

Fig. 1 Schematic diagram of in-house fabricated fluorescence system consisting a 375-nm laser diode, cuvette holder, collimating lens (CL), long pass filter (LPF), cuvette, spectrometer (SM), fibers, USB port, and laptop

lens (UV/Visible collimating lens, 200-2000 nm, Thorlabs) and a LPF (Longpass Filter, Model:FEL0400, Thorlabs) were hooked up. During the measurement, cuvette $(1 \mathrm{~cm} \times 1 \mathrm{~cm} \times 5 \mathrm{~cm})$ filled with saliva was placed inside the sample holder as shown in Fig. 1. Laser light was irradiated onto it through a collimating lens. Fluorescence signals were recorded by the spectrometer (HR 2000, Ocean Optics) at right angle geometry after passing through a 400$\mathrm{nm}$ LPF and CL. To eliminate the specular reflection arising from cuvette and sample holder, LPF was used. For the data acquisition, Ocean Optics Spectra Suit software was utilized. Integration time of $1 \mathrm{~s}$ was fixed and the spectra of saliva were recorded in the scan range of $400-800 \mathrm{~nm}$ in the interval of $0.46 \mathrm{~nm}$. After collection of samples, measurements were taken and completed within an hour. Later on, histopathology reports of biopsy samples were obtained and compared with spectra of SCC and dysplastic patients.

\section{Analysis method}

Receiver operating characteristic (ROC) analysis is a statistical method which is used to evaluate the performance of a diagnostic test. In the ROC analysis, a binary data set (e.g., SCC/normal) is classified by calculating a cut-off value. In ROC analysis, a two-dimensional curve is generated named ROC curve. ROC curve is plot of true-positive rate (sensitivity) along $Y$-axis against false-positive rate (1-specificity) along $X$-axis. Sensitivity and specificity are the fundamental measures of diagnostic accuracy and defined as the probabilities of correctly identifying diseased (cancerous) and healthy (normal) groups as positive and negative respectively. A better performance test has higher values of sensitivity and specificity. Area under the ROC curve (AUC) is also a measure of performance of the test. Larger value of AUC indicates better performance of a given test. AUC value of 1 indicates perfect test (i.e., accuracy $=100 \%$ ) [32-34]. Sensitivity, specificity, and accuracy for a given test can be defined as:

Sensitivity $=\frac{\text { True positive }(T P)}{\text { True positive( } T P \text { ) })+ \text { False negative }(F N)}$,
Specificity $=\frac{\text { True native }(T N)}{\text { True negative }(T N)+\text { False positive }(F P)}$

Accuracy $=\frac{T P+T N}{T P+T N+F P+F N}$

\section{Results and discussion}

\section{Fluorescence spectroscopy}

Figure 2 shows the spectrum of flavin adenine dinucleotide (FAD) and porphrin solutions in the scan range of 450 to $650 \mathrm{~nm}$ and 550 to $720 \mathrm{~nm}$ respectively. Spectrum of FAD solution consists of a broad band near $520 \mathrm{~nm}$ as shown in
Fig. 2 Fluorescence spectrum of FAD and porphyrin solutions in the scan range of 450 to $650 \mathrm{~nm}$ and 550 to $720 \mathrm{~nm}$ (a) FAD spectrum (b) porphyrin spectrum
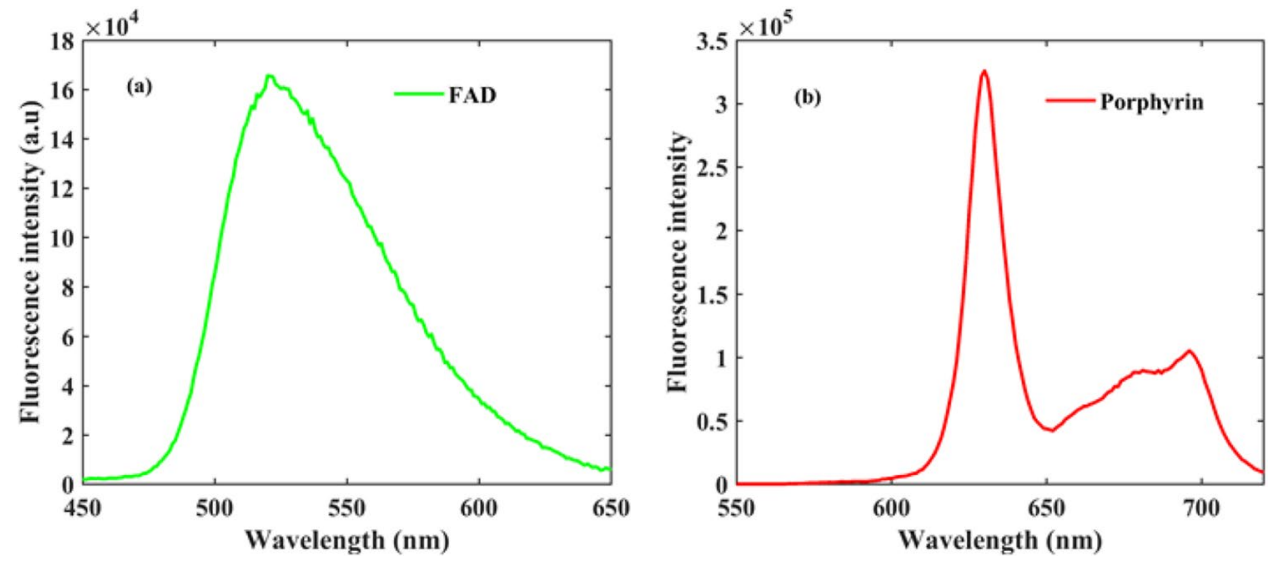
Fig. 2a. Porphyrin spectrum as shown in Fig. $2 \mathrm{~b}$ consists of one major peak at $630 \mathrm{~nm}$ and three minor peaks at 660 , 678 , and $697 \mathrm{~nm}$ respectively. Figure $3 \mathrm{a}$ shows the averaged spectra of SCCpatients $(n=31)$, dysplastic patients $(n=22)$, and normal volunteers $(n=20)$ in the scan range of 400 to $800 \mathrm{~nm}$. It shows a FAD band near $500 \mathrm{~nm}$; porphyrin bands at 634,674 , and $701 \mathrm{~nm}$; and Raman bands at 403 and $437 \mathrm{~nm}$. It can be seen that fluorescence intensities in SCC and dysplastic groups are higher than control group, especially difference between SCCand normal groups is significantly higher. Peak values of intensities of FAD bands for SCC, dysplastic, and control groups are $2658 \pm 1242$, $1840 \pm 765$, and $614 \pm 213$ respectively. Raman peaks present in SCCgroup have low intensity values than the control group. Dysplastic group also display reduced intensity values than the normal but not as significant as SCC group. Reduction of Raman peaks may be due to increase in concentration of saliva samples in diseased groups (SCC and dysplasia) [35]. It was also noticed during the study that diseased groups have higher concentration of saliva than the control group. Presence of porphyrin bands was observed in 25 SCCpatients, 20 dysplastic patients, and 15 control group patients. Intensities of porphyrin bands in SCCand dysplastic cases were significantly higher than the control group. In all the three groups, both 634- and 674-nm bands showed variation in intensities in different samples. Some of the saliva samples of all three groups have displayed dominance of 634-nm band, some have 676-nm band dominance, and few have displayed almost equal counts. In few cases of $\operatorname{SCC}(n=7)$ and dysplastic $(n=3)$ groups, porphyrin bands were dominant over the FAD bands. However, in the control group, FAD bands were always dominant. A typical spectrum of such patients with porphyrin dominance and a spectrum of control group associated with porphyrin band are shown in Fig. 3b. In few cases, difference in band intensities of FAD and porphyrin was very close. It indicates that choosing a particular band or their intensity ratios cannot be a good choice for diagnosis.
It was observed that fluorescence intensities of saliva samples start diminishing with the progress of time (after couple of hours), especially porphyrin band reduction was faster than the FAD band. It was also noted that when saliva sample was poured in the cuvette and spectra were recorded without any delay, fluctuation in fluorescence intensities was observed. It was therefore important to wait for few minutes ( 2 to $3 \mathrm{~min}$ ) and then proceed to record the spectra to get stable data (i.e., free from error).

\section{Data analysis}

Receiver operating characteristic (ROC) analysis has been employed on the area values of the spectra of SCC, dysplasia, and normal. Three spectral ranges $(455-600 \mathrm{~nm}$, 605-770 nm, and 400-800 nm) which correspond to FAD band, porphyrin bands, and combination of all the three bands (Raman, FAD, and porphyrin) have been selected from the spectra of each group and area under these spectral regions is computed. Scatter data plots of area values in the spectral range (400-800 $\mathrm{nm}$ ) among SCC to normal, dysplasia to normal, and SCC to dysplasia are plotted against the number of samples and shown in Fig. 4a-c. ROC analysis is applied on these binary data sets of area values. ROC analysis computes the sensitivity, specificity, accuracy, area under the curves, and cut-off values for the given test. Cut-off values found for the binary data sets of SCC to normal, dysplasia to normal, and SCC to dysplasia are $6 \times 10^{5}, 5 \times 10^{5}$, and $8.6 \times 10^{5}$ respectively and drawn in the scatter data plots as shown by solid, dash, and dash-dot lines respectively. ROC analysis generates the ROC curves which are shown in Fig. 4d. SCC to normal, dysplasia to normal, and SCC to dysplasia are differentiated with sensitivities of $96.77 \%$ (30/31), 81.82\% (18/22), 80.65\% (25/31) and specificities of $100 \%$ (20/20), 95\% (19/20), 81.82\% (18/22) with the overall accuracies of $98 \%$ (50/51), 92.85\% (39/42), and 81.13\% (43/53) respectively. Areas under the ROC curves (AUC) are found to be $0.95,0.89$, and 0.86 respectively. ROC analysis is also applied on the area values of the spectral range
Fig. 3 Fluorescence spectra of SCC, dysplastic, and normal saliva samples in the scan range of 400-800 nm taken in the interval of $0.46 \mathrm{~nm}$ (a) mean spectra (b) typical spectra
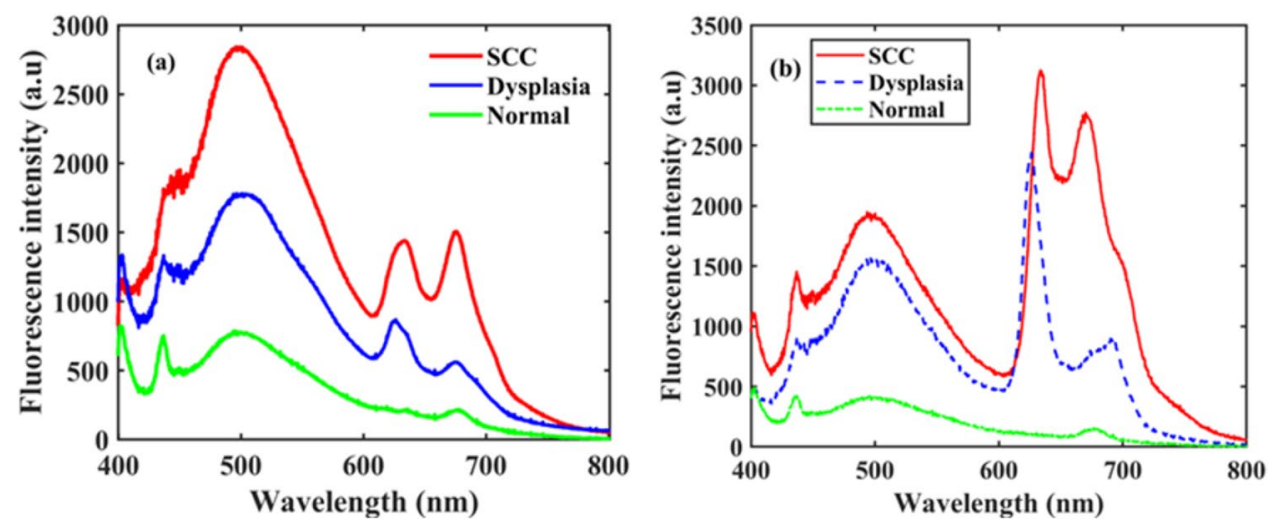
Fig. 4 Scatter data plots among SCC, dysplasia, and normal samples (a) SCC/normal (b) dysplasia/normal (c) SCC to dysplasia (d) ROC curve
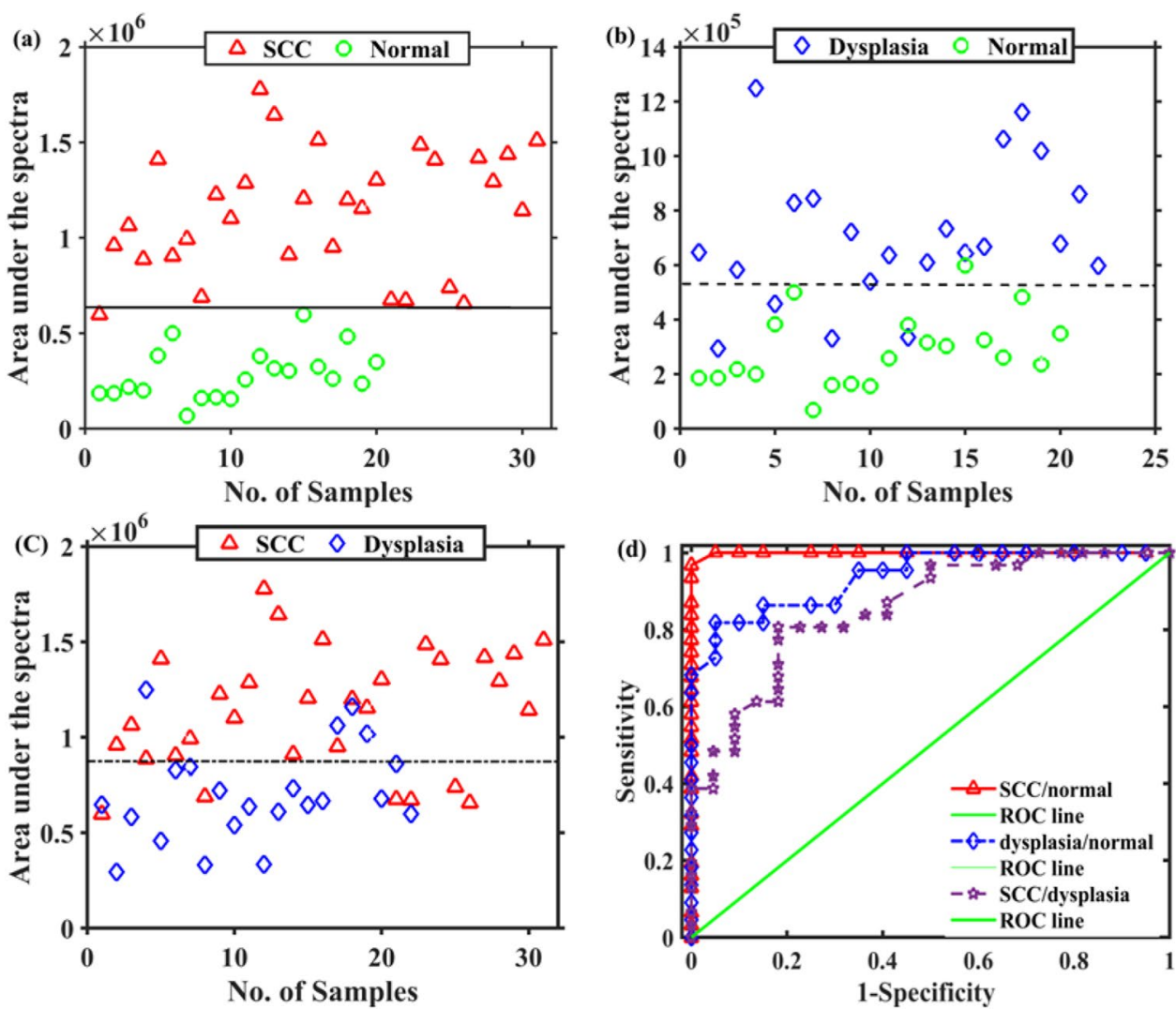

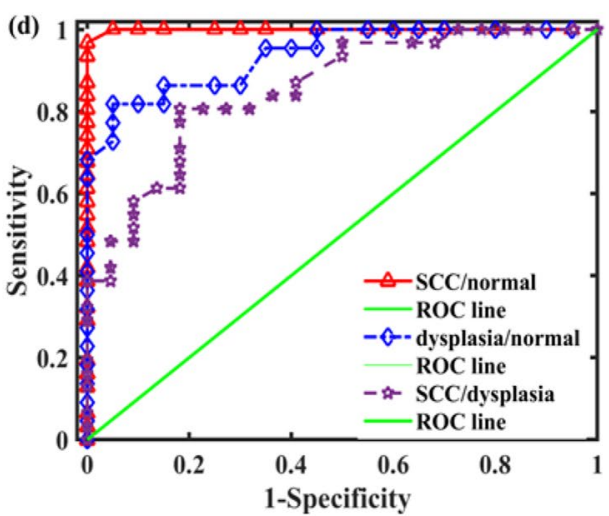

(455-600 nm) and it is able to discriminate the respective groups with sensitivities $90 \%, 72.73 \%, 87 \%$ and specificities $100 \%, 95 \%, 59 \%$ with the overall accuracies of $94 \%$ (48/51), $83 \%$ (35/42), and 75\% (40/53) respectively. However, ROC applied in the spectral range $(605-770 \mathrm{~nm})$ is able to discriminate the respective groups with sensitivities and specificities of $96.77 \%, 72.73 \%$, and $48.39 \%$, and $85 \%, 100 \%$, and $95.45 \%$ with the overall accuracies of $92 \%(48 / 51), 86 \%$ (35/42), and 68\% (36/53) respectively. Obtained ROC curves are shown in Fig. 5a and b respectively. AUC values found for 455 to $600 \mathrm{~nm}$ and 605 to $770 \mathrm{~nm}$ spectral ranges are $0.90,0.84,0.73$ and $0.90,0.86,0.67$ respectively. In differentiating SCC to normal and dysplasia to normal, AUC and accuracy values for the spectral range which correspond to FAD and porphyrin are very close but less than the values of entire spectral range (400-88 nm). It indicates that choosing a particular biomarker or band ratios will not be a good choice for discrimination among the groups. For better comparison, sensitivity, specificity, accuracy, and AUC values from all the three spectral ranges are summarized in Tables 1 and 2 respectively.
Fig. 5 ROC curves among SCC, dysplasia, and normal groups for (a) area values in the spectral range 455-600 $\mathrm{nm}$ and (b) area values in the spectral range 605-770 nm
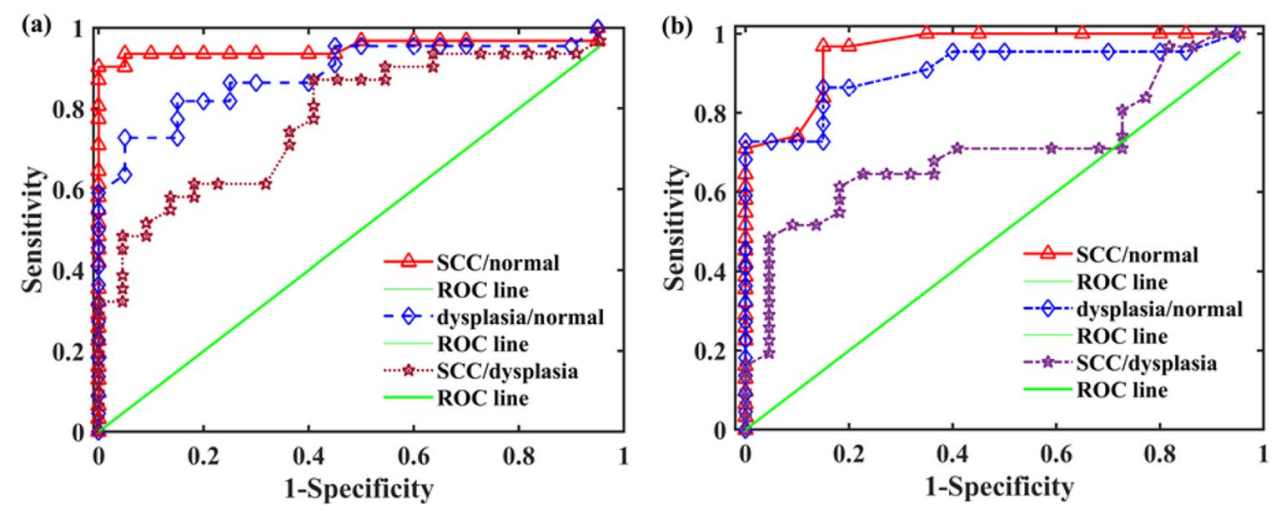
Table 1 Sensitivity and specificity for SCC, dysplastic, and control groups in three different spectral ranges

\begin{tabular}{|c|c|c|c|c|c|c|}
\hline \multirow[t]{2}{*}{ Sample type } & \multicolumn{2}{|c|}{ Spectral range (455-600) } & \multicolumn{2}{|c|}{ Spectral range (605-770) } & \multicolumn{2}{|c|}{ Spectral range (400-800) } \\
\hline & Sensitivity & Specificity & Sensitivity & Specificity & Sensitivity & Specificity \\
\hline SCC/normal & $90 \%$ & $100 \%$ & $97 \%$ & $85 \%$ & $97 \%$ & $100 \%$ \\
\hline Dysplasia/normal & $73 \%$ & $95 \%$ & $73 \%$ & $100 \%$ & $82 \%$ & $95 \%$ \\
\hline SCC/dysplasia & $87 \%$ & $59 \%$ & $48 \%$ & $95 \%$ & $81 \%$ & $82 \%$ \\
\hline
\end{tabular}

Table 2 Accuracy and AUC values among the three groups in three different spectral ranges

\begin{tabular}{|c|c|c|c|c|c|c|}
\hline \multirow[t]{2}{*}{ Sample type } & \multicolumn{2}{|c|}{ Spectral range (455-600) } & \multicolumn{2}{|c|}{ Spectral range (605-770) } & \multicolumn{2}{|c|}{$\begin{array}{l}\text { Spectral range } \\
(400-800)\end{array}$} \\
\hline & Accuracy & AUC & Accuracy & AUC & Accuracy & AUC \\
\hline SCC/normal & $94 \%$ & 0.90 & $92 \%$ & 0.90 & $98 \%$ & 0.95 \\
\hline Dysplasia/normal & $83 \%$ & 0.84 & $86 \%$ & 0.86 & $93 \%$ & 0.89 \\
\hline SCC/dysplasia & $75 \%$ & 0.73 & $68 \%$ & 0.67 & $81 \%$ & 0.86 \\
\hline
\end{tabular}

\section{Conclusion}

In summary, the diagnostic efficacy of fluorescence spectroscopy on human saliva samples collected from three groups SCC, dysplastic, and control of head and neck cancer patients has been investigated. Fluorescence spectra indicate the presence of FAD band and porphyrin bands with significant difference in fluorescence intensities among the three groups. FAD bands were dominant in all the three groups, whereas porphyrin bands were also prominent in SCC and dysplastic groups. ROC analysis applied on the area values of the spectra (400-800 nm) was able to differentiate the groups with higher values of sensitivity and specificity. However, in other two spectral ranges (455-600 $\mathrm{nm}$ and 605-770 nm), sensitivities and specificities were found lower among SCC to dysplasia and dysplasia to normal. Results show that selection of a particular band will not be a good choice, since both FAD and porphyrin bands have shown dominance in the spectra. This study reveals that human saliva may be good diagnostic medium for inaccessible lesions of head and neck cancer. This methodology may also be useful for the detection of coronavirus.

Acknowledgements I would like to acknowledge Prof. Asima Pradhan of the Department of Physics IIT Kanpur and Dr. Surendra Kumar Kanaujia of GSVM Medical College Kanpur. Facilities of Biophotonics lab (SL-111) at IIT Kanpur are used.

Data availability The author has not any objection regarding it.

Code availability The author has used MATLAB software to write the code.

\section{Declarations}

Ethical approval To conduct this study, ethical clearance was taken which was approved by GSVM Medical College Kanpur and IIT Kanpur committee members.

Consent to participate Consent forms from all the participants (patients/volunteers) were taken before collecting the saliva samples.

Consent to publication The author is permitting to publish.

Conflict of interest The author declares no competing interests.

\section{References}

1. Argiris A, Karamouzis MV, Raben D, Ferris RL (2008) Head and neck cancer. Lancet 371:1695-1709

2. Chaturvedi AK, Anderson WF, Tieulent JL et al (2013) Worldwide trends in incidence rates for oral cavity and oropharyngeal cancers. J Clin Oncol 31(36):4550-4559

3. Ferlay J, Soerjomataram I, Dikshit R et al (2015) Cancer incidence and mortality worldwide: sources, methods and major patterns in globocan. Int J Cancer 136(5):359-386

4. Mazul AL, Taylor JM, Divaris K, Weissler MC et al (2017) Oral health and human papillomavirus-associated head and neck squamous cell carcinoma. Cancer 123(1):71-80

5. Scully C, Bagan JV, Hopper C, Epstein JB (2008) Oral cancer: current and future diagnostic techniques. Am J Dent 21(4):199-209

6. Dammann F, Bootz F, Cohnen M, Haßfeld et al (2014) Diagnostic imaging modalities in head and neck disease. Dtsch Arztebl Int 111(23-24):417

7. Omar EA (2015) Current concepts and future of noninvasive procedures for diagnosing oral squamous cell carcinoma-a systematic review. Head Face Med 11:6 
8. Schantz SP, Kolli VS (1998) In vivo cellular fluorescence and histological characteristics of head and neck cancer. Clin Cancer Res 4:1177-1182

9. Inaguma M, Hashimoto K (1999) Porphyrin-like fluorescence in oral cancer. Cancer 86(11):2201-2211

10. De Veld DC, Witjes MJ, Sterenborg HJ, Roodenburg JL (2005) The status of in vivo autofluorescence spectroscopy and imaging for oral oncology. Oral Oncol 41:117-131

11. Teh SK, Zheng W, Lau DP, Huang Z (2009) Spectroscopic diagnosis of laryngeal carcinoma using near-infrared Raman spectroscopy and random recursive partitioning ensemble techniques. Analyst 134:1232-1239

12. DeCoro M, Wilder-Smith P (2010) Potential of optical coherence tomography for early diagnosis of oral malignancies. Expert Rev Anticancer Ther 10(3):321-329

13 Alfano RR (2012) Advances in ultrafast time resolved fluorescence physics for cancer detection in optical biopsy. AIP Adv 2(1):011103

14. Singh SP, Ibrahim O, Byrne HJ, Mikkonen JW et al (2016) Recent advances in optical diagnosis of oral cancers: review and future perspectives. Head Neck 38(1):2403-2411

15. Kumar P, Kanaujia SK, Singh A, Pradhan A (2019) In vivo detection of oral precancer using a fluorescence-based, in-house-fabricated device: a Mahalanobis distance-based classification. Lasers Med Sci 34:1243-1251

16. Delank W, Khanavkar B, Nakhosteen JA, Stool W (2000) A pilot study of autofluorescent endoscopy for the in vivo detection of laryngeal cancer. Laryngoscope 110:368-373

17. Lau DP, Huang Z, Lui H et al (2005) Raman spectroscopy for optical diagnosis in the Larynx: preliminary findings. Lasers Surg Med 37:192-200

18. Harris AT, Rennie A, Waqar-Uddin H, Wheatley SR (2010) Raman spectroscopy in head and neck cancer. Head Neck Oncol 2(1):26

19. Kraft M, Betz CS, Leunig A, Arens C (2011) Value of fluorescence endoscopy for the early diagnosis of laryngeal cancer and its precursor lesions. Head Neck 10(1002):21565

20. Pichardo JL, García OB, Franco MR, Juarez GG (2007) Raman spectroscopy and multivariate analysis of serum samples from breast cancer patients. Lasers Med Sci 22:229-236

21. Harris AT, Lungari A, Needham CJ et al (2009) Potential for Raman spectroscopy to provide cancer screening using a peripheral blood sample. Head Neck 1:34

22. Li Xiaozhou, Yang T, Lin J (2012) Spectral analysis of human saliva for detection of lung cancer using surface-enhanced Raman spectroscopy. J Biomed Opt 17(3):037003
23. Yuvaraj M, Udayakumar K, Jayanth V et al (2014) Fluorescence spectroscopic characterization of salivary metabolites of oral cancer patients. J Photochem Photobiol 130:153-160

24. Wu JY, ChenYi CHR et al (2010) Potential biomarkers in saliva for oral squamous cell carcinoma. Oral Oncol 46:226-231

25. Markopoulos AK, Michailidou EZ, Tzimagiorgis G (2010) Salivary markers for oral cancer detection. Open Den J 4:172-178

26. Soukos NS, Crowley K, Bamberg MP, Gillies R et al (2000) A rapid method to detect dried saliva stains swabbed from human skin using fluorescence spectroscopy. Forensic Sci Int 114:133-138

27. Virkler K, Lednev IK (2009) Analysis of body fluids for forensic purposes: from laboratory testing to non-destructive rapid confirmatory identification at a crime scene. Forensic Sci Int 188:1-17

28. Patil A, Choudhari KS, Unnikrishnan VK et al (2013) Salivary protein markers: a noninvasive protein profile-based method for the early diagnosis of oral premalignancy and malignancy. $\mathbf{J}$ Biomed Opt 18(10):101317

29. Kumar P, Singh A, Kanaujia SK, Pradhan A (2018) Human saliva for oral precancer detection: a comparison of fluorescence and Stoke shift spectroscopy. J Fluoresc 28(1):419-426

30. To KK, Tsang OT, Yip CC, Chan KH et al (2020) Consistent detection of 2019 Novel Coronavirus in saliva. Clin Infect Dis 71(15):841-843

31. Desai S, Mishra SV, Joshi A et al (2020) Raman spectroscopybased detection of RNA viruses in saliva: a preliminary report. J Biophotonics 13(10):e202000189

32. Fawcett T (2006) An introduction to ROC analysis. Pattern Recogn Lett 27:861-874

33. Zou KH, O’Malley AJ, Mauri L (2007) Receiver-operating characteristic analysis for evaluating diagnostic tests and predictive models. Circulation 115(5):654-657

34. Hajian-Tilaki K (2013) Receiver operating characteristic (ROC) curve analysis for medical diagnostic test evaluation. Caspian $\mathrm{J}$ Intern Med 4(2):627

35. Nagler R, Bahar G, Shpitzer T, Feinmesser R (2006) Concomitant analysis of salivary tumor markers - a new diagnostic tool for oral cancer. Clin Cancer Res 12(13):3979-3984

Publisher's note Springer Nature remains neutral with regard to jurisdictional claims in published maps and institutional affiliations. 